\title{
Exponential Stability of Nonlinear Differential Repetitive Processes with Applications to Iterative Learning Control ${ }^{\star}$
}

\author{
Berk Altin ${ }^{\mathrm{a}}$, Kira Barton ${ }^{\mathrm{b}}$ \\ ${ }^{a}$ Department of Computer Engineering, University of California, Santa Cruz, CA 95064 \\ ${ }^{\mathrm{b}}$ Department of Mechanical Engineering, University of Michigan, Ann Arbor, MI 48109
}

\begin{abstract}
This paper studies exponential stability properties of a class of two-dimensional (2D) systems called differential repetitive processes (DRPs). Since a distinguishing feature of DRPs is that the problem domain is bounded in the "time" direction, the notion of stability to be evaluated does not require the nonlinear system defining a DRP to be stable in the typical sense. In particular, we study a notion of exponential stability along the discrete iteration dimension of the $2 \mathrm{D}$ dynamics, which requires the boundary data for the differential pass dynamics to converge to zero as the iterations evolve. Our main contribution is to show, under standard regularity assumptions, that exponential stability of a DRP is equivalent to that of its linearized dynamics. In turn, exponential stability of this linearization can be readily verified by a spectral radius condition. The application of this result to iterative learning control (ILC) is discussed. Theoretical findings are supported by a numerical simulation of an ILC algorithm.
\end{abstract}

Key words: Recursive control algorithms; Lyapunov stability; nonlinear systems; learning control; iterative methods.

\section{Introduction}

For recursive nonlinear systems in the explicit form

$$
\left\{\begin{array}{l}
\dot{x}_{k+1}(t)=f\left(x_{k+1}(t), y_{k}(t), t\right), \\
y_{k+1}(t)=g\left(x_{k+1}(t), y_{k}(t), t\right),
\end{array}\right.
$$

where $(t, k) \in[0, T] \times\{0,1, \ldots\}$ for some $T \in[0, \infty)$, we are interested in finding necessary and sufficient conditions that establish local exponential stability. The vectors $x_{k}(t) \in \mathbb{R}^{n}$ and $y_{k}(t) \in \mathbb{R}^{m}$ of this model represent the state and output, respectively. To uniquely determine the solution of (1), it will be necessary to specify boundary conditions $y_{0}$ and $\mathbf{x}(0) \triangleq\left\{x_{k+1}(0)\right\}_{k=0}^{\infty}$.

Roughly speaking, the notions of stability to be studied throughout this paper will be weak, in the sense that they will not require the one-dimensional (1D) control system given by $f$ to be stable. For example, exponential stability of (1) will imply that the function sequence $\left\{y_{k}\right\}_{k=0}^{\infty}$ converges exponentially to zero in an

\footnotetext{
* This paper was not presented at any IFAC meeting. Corresponding author B. Altın. Tel. +1 (831) 459-2939.

Email addresses: berkaltin@ucsc.edu (Berk Altın), bartonkl@umich.edu (Kira Barton).
}

Preprint submitted to Automatica appropriate signal norm, provided the boundaries are small, and $\mathbf{x}(0)$ also converges exponentially to zero. The precise meaning of stability for this class of systems will be defined later in Section 2.

The nonlinear system (1) appears in many practical problems of interest and falls into the larger class of twodimensional (2D) dynamic systems called repetitive (or multipass, earlier in the literature) processes ${ }^{1}$, in which information propagation occurs along two axes of independent variables. These processes are characterized by a sequence of passes with finite length that act as forcing functions on the dynamics of future passes (Rogers et al. 2007): The output solution sequence $\left\{y_{k}\right\}_{k=0}^{\infty}$ of (1) can be found by applying the nonlinear system with differential dynamics described by the functions $f$ and $g$ in a repetitive manner. Hence, we will call any system of the form (1) a differential repetitive process (DRP). The counterpart of the DRP (1) in the broader 2D systems theory, where it is assumed that $T=\infty$, will be called a 2D mixed continuous-discrete time system.

The repetitive process paradigm arises in the modeling of certain engineering applications such as long wall

1 Not to be confused with repetitive control. 
coal cutting (Edwards 1974) and metal rolling (Foda and Agathoklis 1992, Edwards and Owens 1982). A rich set of examples to these systems can also be found on a more abstract level since recursive algorithms for 1D dynamic systems can be treated as repetitive processes; e.g. iterative solutions to nonlinear optimal control problems (Zidek and Kolmanovsky 2015, Gupta et al. 2013), nonlinear inversion methods (Devasia et al. 1996), iterative estimation and control design (Albertos and Sala 2002), or the constructive proof of the Picard-Lindelöf theorem. A well-known class of algorithms that can be expressed in the repetitive process framework is iterative learning control (ILC) (Kurek and Zaremba 1993, Hladowski et al. 2010, Ahn et al. 2007), wherein the inverse image of a desired output under a 1D input-output system is constructed through a recurrence relation inducing pass-to-pass dynamics. This problem will be tackled in Section 5.

The study of DRPs and other 2D systems bearing similarities with (1) has a long history, beginning with the Roesser and Fornasini-Marchesini models introduced in the 1970s (Roesser 1975, Fornasini and Marchesini 1976, Fornasini and Marchesini 1978). In particular, stability and performance properties of DRPs and 2D mixed continuous-discrete time systems, along with corresponding control strategies, have been researched extensively, predominantly for linear timeinvariant (LTI) systems-see (Rogers et al. 2007, Chesi and Middleton 2014, Chesi and Middleton 2015) and references therein. On the other hand, the need to develop rigorous stability tests in the nonlinear systems context has been highlighted only very recently. Among these works, (Yeganefar et al. 2013) presents forward and converse Lyapunov theorems for nonlinear Roesser models, with extensions to the stochastic case given in (Pakshin et al. 2011), and a 2D Lyapunov function approach is employed to prove exponential stability of DRPs in (Emelianov et al. 2014). It is also worth noting that the DRP (1) can be viewed as an infinite-dimensional hybrid system (Liu and Teel 2016, Barreiro and Baos 2010, Sun et al. 2005) by concatenating the passes; e.g. by letting $x(\tau, k+1) \triangleq x_{k+1}(t)$ with $\tau=t+k T$, subject to the periodic reset $x(k T, k+1)=x_{k+1}(0)$, where $T$ plays the role of an inherent delay, $\tau$ the ordinary time, and $k$ the jump time/index. As this reset function would change based on the prespecified boundary condition $\mathbf{x}(0)$ and lacks any other structure, we will not follow a hybrid systems approach in the ensuing analysis. See also (Rogers et al. 2007) for DRP modeling of a class of delay differential equations.

The objective of this paper is to contribute to the recent literature on nonlinear repetitive process and 2D systems literature, and provide a connection between nonlinear DRPs of the form (1) and their linear counterparts. Therefore, our aim is to certify local exponential stability of the DRPs via an appropriate linearization of (1), and establish an analogue of the classical result

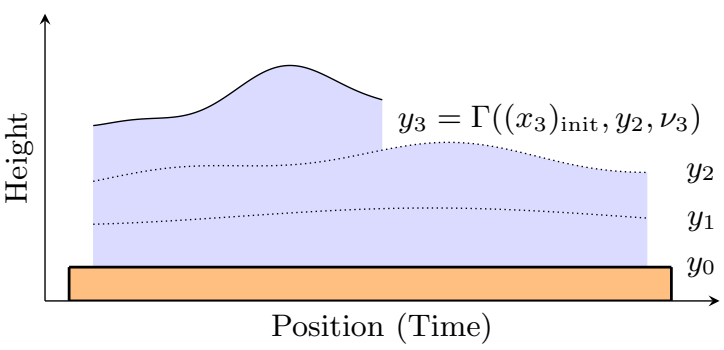

Fig. 1. AM systems as repetitive processes: The substrate topography determines the initial output $y_{0}$. The operator $\Gamma$ maps the initial state $\left(x_{3}\right)_{\text {init }}$ and input $\nu_{3}$ of pass 3 (in-layer dynamics), along with the prior pass profile $y_{2}$ (layer-to-layer dynamics), to pass profile $y_{3}$. The layer-to-layer dynamics is affected by physical phenomena such as material curing.

that exponential stability of a 1D system is equivalent to that of its linear approximation, thereby expanding on the findings of (Altin and Barton 2015). Our primary motivation for this study comes from additive manufacturing (AM) systems, wherein material in the fluid phase is often deposited in a layer-by-layer fashion (Fig. 1), leading to 2D dynamics: For instance, the laser metal deposition (LMD) process is characterized by 1D (inlayer) dynamics that are height dependent due to heat transfer from prior layers (Sammons et al. 2013). It is possible to achieve accurate material distribution for the LMD process via linear repetitive process control techniques and a more control-oriented model consisting of static nonlinearities. This, however, requires the implicit assumption that the controlled nonlinear process is $l o-$ cally stable around its linearized equilibrium (Sammons et al. 2014). As a secondary motivation, in the ILC literature, it has been noted that nonlinear update laws have not been researched, save for adaptive laws for locally Lipschitz plants, and a systematic theory of nonlinear ILC is an open question ( $\mathrm{Xu}$ 2011, Moore et al. 2006).

The rest of the paper is organized as follows: Section 2 introduces the necessary background, establishes the key Lipschitz property of the nonlinear operator, and states formal stability definitions. Stability theory for LTI systems is extended to the linear time-varying (LTV) case in Section 3. Our main result, which establishes equivalence in terms of exponential stability between a DRP and its linearization, is presented in Section 4. Applications of this result to exponential stability analysis of ILC is discussed in Section 5. An illustrative example is given in Section 6 through an ILC system. Concluding remarks are given in Section 7. In the hope of improving readability of the paper, the proof of Proposition 14 is given in Appendix A. Proofs of certain immediate technical results are omitted for brevity and can be found in (Altin and Barton 2017). 


\section{Background and Preliminaries}

This section will introduce the background material pertinent to our analysis, and lay out stability definitions for the DRP (1). The precise definitions of stability to be presented will show the crucial difference between DRPs and 2D mixed continuous-discrete time systems, as the latter studies the trajectory of the real vector $y_{k}(t)$ over $\{0,1, \ldots\} \times[0, \infty)$. In linear repetitive process theory, the gap between these two classes of systems is bridged via the stronger notion of stability along the pass (Rogers et al. 2007), which requires the stability parameters to be $T$ independent. Although this property is desirable in experimental implementations or numerical simulations, we will forgo this requirement for theoretical purposes.

Notation: We use $\mathbb{R}$ to represent real numbers, $\mathbb{N}$ nonnegative integers, and $\mathbb{C}$ complex numbers. The spectral radius of a linear operator is denoted by $\rho($.$) . The$ identity and zero operators are denoted $I$ and 0 , respectively. For a real vector, $\|$.$\| denotes any of the equiva-$ lent norms in $\mathbb{R}^{p}$. $\mathcal{L}_{p}$ is the space of Lebesgue measurable functions on the compact interval $[0, T]$ with finite $\mathcal{L}_{p}$ norm, $p \in[1, \infty]$. The space of all sequences on $\mathbb{R}^{p}$ which converge to 0 is denoted $c_{0}$.

The inequalities below, stated without proof, will be of use for convergence analysis. Note that the convergence parameters $2 /(1-a) \geq 1$ and $(1+a) / 2 \in(0,1)$ are continuous increasing functions of $a$ on $(0,1)$.

Claim 1 Let $\mathbf{a} \triangleq\left\{a_{k+1}\right\}_{k=0}^{\infty}$ and $\mathbf{b} \triangleq\left\{b_{k+1}\right\}_{k=1}^{\infty}$ be real nonnegative sequences, where $\mathbf{b}$ is bounded. Suppose that $a_{k+1}=r a_{k}+b_{k+1}$ for some $r \in(0,1)$ for all $k \in \mathbb{N}$. Then, $\lim \sup _{k \rightarrow \infty} a_{k} \leq(1 /(1-r)) \lim \sup _{k \rightarrow \infty} b_{k}$, and therefore $\mathbf{b} \in c_{0}$ implies $\mathbf{a} \in c_{0}$.

Claim 2 Let $a \in(0,1)$. Then the sequence $\left\{k a^{k-1}\right\}_{k=0}^{\infty}$ is exponentially convergent and

$$
k a^{k-1} \leq \frac{2}{1-a}\left(\frac{1+a}{2}\right)^{k}, \quad \forall k \in \mathbb{N}
$$

\subsection{The Nonlinear Operator over the Finite Horizon}

Before proceeding with further analysis, we will look at the properties of the system (1) as an input-state and input-output operator over the time interval $[0, T]$ : Interchanging $y_{k}$ with $u, x_{k+1}$ with $\chi$, and $y_{k+1}$ with $w$, we consider

$$
\left\{\begin{array}{l}
\dot{\chi}(t)=f(\chi(t), u(t), t), \\
w(t)=g(\chi(t), u(t), t),
\end{array}\right.
$$

for all $t \in[0, T]$. The input $u$ resides in $\mathcal{Y}$, the space of continuously differentiable functions on $[0, T]$. We will impose the following standing assumptions on the nonlinear operator $\Gamma$ that maps the pair $(\chi(0), u)$ to $\chi$ and $w$ :

Assumption 3 The nonlinear system (2) satisfies the following conditions:

(1) The functions $f$ and $g$ vanish at the origin uniformly in time. That is, $f(0,0, t)=0$ and $g(0,0, t)=0$ for all $t \in[0, T]$.

(2) There exists $\delta>0$ such that for every $(\chi(0), u)$ that satisfies $\|\chi(0)\|+\|u\|_{\mathcal{L}_{\infty}}<\delta$, there is a unique integral curve $\chi$ of $(2)$, and $\chi(t)$ is contained in a bounded open connected set $X$ for all $t \in[0, T]$.

(3) There exists a compact set $Y \subset \mathbb{R}^{m}$ that contains the origin in its interior such that $f$ and $g$ are continuously differentiable in $Z \triangleq \operatorname{cl}(X) \times Y \times[0, T]$, where $\operatorname{cl}(X)$ is the closure of $X$.

Assumption 3 is a mild constraint on the system that bypasses the stability requirement in the time domain. We note that since 0 is an equilibrium of the differential equation, the set $X$ must contain the origin. Without loss of generality, we will also assume that $\delta$ is small enough so that $\chi(0) \in X$ and $u(t) \in Y$ for all $t \in[0, T]$ when $\|\chi(0)\|+\|u\|_{\mathcal{L}_{\infty}}<\delta$. We denote by $\Gamma_{x}$ the mapping $(u, \chi(0)) \mapsto \chi$, and by $\Gamma_{y}$ the mapping $(u, \chi) \mapsto w$, SO

$$
(w, \chi)=\Gamma(u, \chi(0)) \triangleq\left(\Gamma_{y}\left(u, \Gamma_{x}(u, \chi(0))\right), \Gamma_{x}(u, \chi(0))\right) .
$$

Now, we can show Lipschitz continuity of the operator $\Gamma$ in the uniform norm topology. See (Altin and Barton 2017) for a proof of this result.

Lemma 4 The nonlinear operator $\Gamma$ given by (2) is locally Lipschitz with respect to $(\chi(0), u)$. That is, there exist positive constants $\bar{\delta}$ and $L$ such that if

$$
\left(w_{i}, \chi_{i}\right) \triangleq \Gamma\left(\chi_{i}(0), u_{i}\right) \text { and }\left\|\chi_{i}(0)\right\|+\left\|u_{i}\right\|_{\mathcal{L}_{\infty}}<\bar{\delta}
$$

where $i \in\{1,2\}$, then

$$
\begin{aligned}
\left\|\chi_{1}-\chi_{2}\right\|_{\mathcal{L}_{\infty}} & \leq L\left(\left\|u_{1}-u_{2}\right\|_{\mathcal{L}_{\infty}}+\left\|\chi_{1}(0)-\chi_{2}(0)\right\|\right) \\
\left\|w_{1}-w_{2}\right\|_{\mathcal{L}_{\infty}} & \leq L\left(\left\|u_{1}-u_{2}\right\|_{\mathcal{L}_{\infty}}+\left\|\chi_{1}(0)-\chi_{2}(0)\right\|\right)
\end{aligned}
$$

\subsection{Boundary Dependent Stability Definitions}

We will now lay out definitions of stability for DRPs. First, we need the following norm to characterize exponential initial state sequences for exponential stability, which is similar to the conventional time-weighted norm used in the ILC literature:

Definition 5 Let $\mathbf{b} \triangleq\left\{b_{k+1}\right\}_{k=0}^{\infty}$ be a sequence on $\mathbb{R}^{p}$. For any $\lambda \in(0,1]$, the exponential $\lambda\left(e_{\lambda}\right)$ norm of $\mathbf{b}$ is defined as $\|\mathbf{b}\|_{e_{\lambda}} \triangleq \sup _{k \in \mathbb{N}} \lambda^{-k}\left\|b_{k+1}\right\|$. 
We leave it to the reader to verify that $e_{\lambda}$, the vector space of all sequences on $\mathbb{R}^{p}$ with finite $e_{\lambda}$ norm, i.e. the space of sequences on $\mathbb{R}^{p}$ that converge geometrically to 0 with rate faster than or equal to $\lambda$, satisfies $e_{\lambda} \subset c_{0} \subset e_{1} \equiv \ell_{\infty}$, for all $\lambda \in(0,1)$. The $e_{\lambda}$ norm also satisfies 1) the shift property, $\left\|\mathbf{b}_{\kappa}\right\|_{e_{\lambda}} \leq \lambda^{\kappa}\|\mathbf{b}\|_{e_{\lambda}}$, where $\mathbf{b}_{\kappa} \triangleq\left\{b_{k+1}\right\}_{k=\kappa}^{\infty}$, given any $\kappa \in \mathbb{N}$, and 2) the $\lambda$ property $\|\cdot\|_{e_{\lambda_{2}}} \leq\|\cdot\|_{e_{\lambda_{1}}}$ when $0<\lambda_{1} \leq \lambda_{2} \leq 1$.

Definition 6 The origin of the DRP (1) is said to be

(1) (Lyapunov) stable, if for all $\epsilon>0$ there exists a scalar $\delta_{1} \in(0, \epsilon)$ such that $\left\|y_{0}\right\|_{\mathcal{L}_{\infty}}+\|\mathbf{x}(0)\|_{e_{1}}<\delta_{1}$ implies $\left\|y_{k}\right\|_{\mathcal{L}_{\infty}}<\epsilon$, for all $k \in \mathbb{N}$,

(2) asymptotically stable, if it is Lyapunov stable and there exists $\delta_{2}>0$ such that $\left\|y_{0}\right\|_{\mathcal{L}_{\infty}}+\|\mathbf{x}(0)\|_{e_{1}}<\delta_{2}$ and $\mathbf{x}(0) \in c_{0}$ implies $\left\|y_{k}\right\|_{\mathcal{L}_{\infty}} \rightarrow 0$,

(3) exponentially stable, if it is asymptotically stable, and there exists $\delta_{3}>0$ and continuous increasing functions $K:(0,1) \rightarrow[1, \infty), \gamma:(0,1) \rightarrow(0,1)$, such that $\left\|y_{0}\right\|_{\mathcal{L}_{\infty}}+\|\mathbf{x}(0)\|_{e_{\lambda}}<\delta_{3}$ implies

$$
\left\|y_{k}\right\|_{\mathcal{L}_{\infty}} \leq K(\lambda) \gamma(\lambda)^{k}\left(\left\|y_{0}\right\|_{\mathcal{L}_{\infty}}+\|\mathbf{x}(0)\|_{e_{\lambda}}\right)
$$

for all $k \in \mathbb{N}$ and $\lambda \in(0,1)$.

In the rest of the paper, since the origin is the only equilibrium of interest, we will simply say that the DRP (1) is (Lyapunov)/asymptotically/exponentially stable. In addition, we will say that the DRP (1) is globally asymptotically (exponentially) stable if $\delta_{2}\left(\delta_{2}\right.$ and $\left.\delta_{3}\right)$ can be chosen to be arbitrarily large. A salient feature of the exponential stability definition above is the dependency of the performance on the convergence speed $\lambda$ of $\mathbf{x}(0)$, expressed via the functions $K$ and $\gamma$, which are continuous and increasing to be physically meaningful. In addition, since 0 is an equilibrium solution for (2), which is Lipschitz with respect to $(\chi(0), u)$ by Lemma 4 , it is straightforward to show that the stability notions above translate directly to the state trajectory.

We will also be considering the case $\mathbf{x}(0)=0$. We will refer to any such DRP as a zero initial states (0-i.s.) system or process. The 0-i.s. system will be defined to be Lyapunov, asymptotically, or exponentially stable if the notions defined above hold for the case of $\mathbf{x}(0)=0$; obviously the 0 -i.s. system is (asymptotically/exponentially) stable if the actual system is (asymptotically/exponentially) stable. Note that (3) is necessary and sufficient for 0 -i.s exponential stability.

\section{Stability of LTV Differential Processes}

In this section, we will focus on systems where $f$ and $g$ are linear with respect to their first two arguments for fixed $t \in[0, T]$, and relax the continuous differentiability assumption to that of continuity; i.e. we will look at LTV differential processes of the form

$$
\left\{\begin{array}{l}
\dot{x}_{k+1}(t)=A(t) x_{k+1}(t)+B(t) y_{k}(t), \\
y_{k+1}(t)=C(t) x_{k+1}(t)+D(t) y_{k}(t),
\end{array}\right.
$$

for all $(t, k) \in[0, T] \times \mathbb{N}$, where $A, B, C, D$ are continuous real matrices of appropriate size.

\subsection{0-i.s Stability and the Spectral Radius}

Similar to the nonlinear case, given the LTV system described by the quadruple $(A, B, C, D)$, we denote by $G_{x}$ the state response to the input and the initial condition, and by $G_{y}$ the mapping from the input and the state to the output. The LTV operator $G$ is defined so that

$(w, \chi)=G(u, \chi(0))=\left(G_{y}\left(u, G_{x}(u, \chi(0))\right), G_{x}(u, \chi(0))\right)$,

and the 0 -i.s. output response $G_{0}(.) \triangleq \pi_{y}(G(., 0))$, where $\pi_{y}$ is the standard projection onto $\mathcal{Y}$. We will first consider the 0-i.s. system described by the discrete system $y_{k+1}=G_{0} y_{k}$ on $\mathcal{Y}$. We have the following claim about $G_{0}$ :

Claim 7 The operator $G_{0}$ is bounded in $\mathcal{L}_{p}$, for any $p \in[1, \infty]$.

Claim 7 makes intuitive sense since linear systems do not have finite escape time. The formal proof of this argument relies on the continuity of state matrices (and hence that of the state-transition matrix) and (Vidyasagar 2002, Theorem 75); see (Altın and Barton 2017). As such, we will expand the space $\mathcal{Y}$ to $\mathcal{L}_{\infty}$, and more generally $\mathcal{L}_{p}$. The stability problem is relatively simple for linear systems as expected: Exponential stability can be conveniently evaluated by the following spectral radius condition, which can easily be proven by Gelfand's spectral radius formula $\rho\left(G_{0}\right)=\lim _{k \rightarrow \infty}\left\|G_{0}^{k}\right\|_{\mathcal{L}_{p}}^{1 / k}($ Weiss 1989):

Theorem 8 The 0-i.s. linear system (4) is exponentially stable (in $\mathcal{L}_{p}$ ) if and only if $\rho\left(G_{0}\right)<1$.

Remark 9 In general, the condition $\rho\left(G_{0}\right)<1$ is sufficient for asymptotic stability, whereas $\rho\left(G_{0}\right) \leq 1$ is necessary (Przyluski 1980). This issue is circumvented in [page 44] (Rogers et al. 2007) by requiring asymptotic stability to be a local property around a nominal operator.

\subsection{Computation of the Spectral Radius}

The computation of the spectral radius will be similar to the procedure outlined for the time-invariant case in (Rogers et al. 2007). Let $P_{z}(t) \triangleq z I-D(t)$, 
where $z \in \mathbb{C}$. It is easy to see that the operator $z I-G_{0}$ mapping $u$ to $\eta$, given by

$$
\left\{\begin{array}{l}
\dot{\chi}(t)=A(t) \chi(t)+B(t) u(t) \\
\eta(t)=-C(t) \chi(t)+P_{z}(t) u(t)
\end{array}\right.
$$

for all $t \in[0, T]$, is invertible if $|z|>\alpha \triangleq \sup _{t \in[0, T]} \rho(D(t))$. In addition, $\left(z I-G_{0}\right)^{-1}$ is bounded (in $\mathcal{L}_{p}$ ) by the bounded inverse theorem. Hence, $\rho\left(G_{0}\right) \leq \alpha$.

Otherwise, given any $\epsilon>0$, let $z \in \mathbb{C}$ be a number such that $P_{z}(t)$ is singular for some $t \in[0, T)$ and $|z|>\alpha-\epsilon$. Such a $z$ exists since the spectral radius of $D$ varies continuously. Define $s \triangleq \min \left\{t \in[0, T]: \operatorname{det}\left(P_{z}(t)\right)=0\right\}$, and set $\eta(t)=\phi \mathbf{1}(t-s)$, where $\phi$ is orthogonal to the range of $P_{z}(s)$, and $\mathbf{1}($.$) is the Heaviside step function.$ Then, one can show by contradiction that there exists no $u \in \mathcal{L}_{\infty}$ that achieves $\eta$ almost everywhere (Altin and Barton 2017). It follows that $z I-G_{0}$ is not surjective. Therefore, $\rho\left(G_{0}\right)=\alpha$.

\subsection{Stability under Nonzero Initial States}

Let $H(.) \triangleq \pi_{y}(G(0,)$.$) be the natural response of the$ LTV system to initial conditions. Then the solution of (4) can be given as

$$
y_{k}=G_{0}^{k} y_{0}+\sum_{i=1}^{k} G_{0}^{k-i} H x_{i}(0), \quad \forall k \in \mathbb{N} .
$$

Now if $\rho\left(G_{0}\right)<1$, by Gelfand's spectral radius formula, there exist scalars $M>0$ and $\zeta \in(0,1)$ such that $\left\|G_{0}^{k}\right\|_{\mathcal{L}_{\infty}} \leq M \zeta^{k}$ for all $k \in \mathbb{N}$. Therefore,

$$
\left\|y_{k}\right\|_{\mathcal{L}_{\infty}} \leq M\left(\zeta^{k}\left\|y_{0}\right\|_{\mathcal{L}_{\infty}}+\|H\|_{\mathcal{L}_{\infty}} \sum_{i=1}^{k} \zeta^{k-i}\left\|x_{i}(0)\right\|\right),
$$

for all $k \in \mathbb{N}$, where $H$ is bounded due to the finitetime assumption ${ }^{2}$. When $\|\mathbf{x}(0)\|_{e_{1}}<\infty$, it is easy to bound the right-hand side of (7) as a linear function of $\left(\left\|y_{0}\right\|_{\mathcal{L}_{\infty}}+\|\mathbf{x}(0)\|_{e_{1}}\right)$. Therefore, the LTV system is stable. Now assume in addition that $\mathbf{x}(0) \in c_{0}$, and consider the partial sum in the second term of the right hand side of $(7), S_{k} \triangleq \sum_{i=1}^{k} \zeta^{k-i}\left\|x_{i}(0)\right\| \geq 0$, for all $k \in \mathbb{N}$. Then, it is easy to verify $S_{k+1}=\zeta S_{k}+\left\|x_{k+1}(0)\right\| \geq 0$ for all $k \in \mathbb{N}$, so by Claim $1, S_{k} \rightarrow 0$. Therefore, we can conclude by (7) that $y_{k} \rightarrow 0$ if $\mathbf{x}(0) \in c_{0}$ and $\rho(G)<1$.

Finally, consider the case $\mathbf{x}(0) \in e_{\lambda}$ for some $\lambda \in(0,1)$.

\footnotetext{
${ }^{2}$ See the discussion of Claim 7 .
}

From (7)

$$
\begin{aligned}
\left\|y_{k}\right\| & \leq M\left(\zeta^{k}\left\|y_{0}\right\|_{\mathcal{L}_{\infty}}+\|H\|_{\mathcal{L}_{\infty}}\|\mathbf{x}(0)\|_{e_{\lambda}} \sum_{i=1}^{k} \zeta^{k-i} \lambda^{i-1}\right) \\
& \leq M\left(\zeta^{k}\left\|y_{0}\right\|_{\mathcal{L}_{\infty}}+\|H\|_{\mathcal{L}_{\infty}}\|\mathbf{x}(0)\|_{e_{\lambda} k \bar{\lambda}^{k-1}}\right)
\end{aligned}
$$

where $\bar{\lambda} \triangleq \max \{\zeta, \lambda\}$, so by Claim 2

$$
\begin{aligned}
& \left\|y_{k}\right\| \leq M \zeta^{k}\left\|y_{0}\right\|_{\mathcal{L}_{\infty}} \\
& \quad+M\|H\|_{\mathcal{L}_{\infty}}\|\mathbf{x}(0)\|_{e_{\lambda}} \frac{2}{1-\bar{\lambda}}\left(\frac{1+\bar{\lambda}}{2}\right)^{k}
\end{aligned}
$$

and since $\zeta \leq \bar{\lambda}<(1+\bar{\lambda}) / 2<1$,

$$
\begin{aligned}
& \left\|y_{k}\right\|_{\mathcal{L}_{\infty}} \leq \overbrace{M \max \left\{1, \frac{2\|H\|_{\mathcal{L}_{\infty}}}{1-\bar{\lambda}}\right\}}^{K_{G}(\bar{\lambda})} \\
& \times(\underbrace{(1+\bar{\lambda}) / 2}_{\gamma_{G}(\bar{\lambda})})^{k}\left(\left\|y_{0}\right\|_{\mathcal{L}_{\infty}}+\|\mathbf{x}(0)\|_{e_{\lambda}}\right), \quad \forall k \in \mathbb{N} .
\end{aligned}
$$

Noting that $K_{G}(\max \{\zeta, \lambda\})$ and $\gamma_{G}(\max \{\zeta, \lambda\})$ defined in (8) are both continuous and increasing in $\lambda$ on $(0,1)$, we can conclude the system to be exponentially stable. With this, our findings can be summarized as follows:

Theorem 10 For the LTV DRP (4), the following are equivalent:

(1) The DRP (4) is globally exponentially stable.

(2) The 0-i.s. DRP (4) is globally exponentially stable.

(3) The condition $\max _{t \in[0, T]} \rho(D(t))<1$ holds.

Remark 11 The analysis of Section 3.3 extends to any $\mathcal{L}_{p}$ norm since $\rho\left(G_{0}\right) \leq \alpha$ for all $p \in[1, \infty]$. Therefore, $\alpha<1$ implies global exponential stability in $\mathcal{L}_{p}$.

\section{Linearized Stability of DRPs}

We will now establish the equivalence between exponential stability of a nonlinear DRP of the form (1) with that of its linearization. The linearization of (1) will mirror that of the $1 \mathrm{D}$ case, in other words, we will be linearizing the differential operator (2) as is typical in feedback control. This will be done as follows: Since $f$ and $g$ are continuously differentiable,

$$
\left\{\begin{array}{l}
\dot{\chi}(t)=\bar{A}(t) \chi(t)+\bar{B}(t) u(t)+b(\chi(t), u(t), t), \\
w(t)=\bar{C}(t) \chi(t)+\bar{D}(t) u(t)+d(\chi(t), u(t), t),
\end{array}\right.
$$


for some continuous functions $b$ and $d$, as

$$
\begin{array}{ll}
\bar{A}(t) \triangleq \frac{\partial f}{\partial \chi}(0,0, t), \quad \bar{B}(t) \triangleq \frac{\partial f}{\partial u}(0,0, t), \\
\bar{C}(t) \triangleq \frac{\partial g}{\partial \chi}(0,0, t), \quad \bar{D}(t) \triangleq \frac{\partial g}{\partial u}(0,0, t),
\end{array}
$$

are continuous. Consequently, the linearization of (1) will be defined as the following 2D system:

$$
\left\{\begin{array}{l}
\dot{\bar{x}}_{k+1}(t)=\bar{A}(t) \bar{x}_{k+1}(t)+\bar{B}(t) \bar{y}_{k}(t), \\
\bar{y}_{k+1}(t)=\bar{C}(t) \bar{x}_{k+1}(t)+\bar{D}(t) \bar{y}_{k}(t),
\end{array}\right.
$$

for all $(t, k) \in[0, T] \times \mathbb{N}$, with boundary conditions satisfying $\overline{\mathbf{x}}(0)=\mathbf{x}(0)$ and $\bar{y}_{0}=y_{0}$.

\subsection{Asymptotics of the Nonlinear Perturbations}

Let $f_{i}$ be the $i$-th output of $f$. Since $f$ is continuously differentiable in $Z$ and $f(0,0, t)=0$, by the multivariable mean value theorem, there exists a point $\left(\xi_{i}^{*}, v_{i}^{*}\right)$ on the line segment connecting $(\xi, v)$ to the origin such that

$$
f_{i}(\xi, v, t)=\left[\frac{\partial f_{i}}{\partial \xi}\left(\xi_{i}^{*}, v_{i}^{*}, t\right) \frac{\partial f_{i}}{\partial v}\left(\xi_{i}^{*}, v_{i}^{*}, t\right)\right]\left[\begin{array}{l}
\xi \\
v
\end{array}\right]
$$

in a neighborhood of $0 \in \mathbb{R}^{n} \times \mathbb{R}^{m}$. Equivalently,

$$
\begin{aligned}
& f_{i}(\xi, v, t)=\left[\begin{array}{ll}
\bar{A}_{i}(t) & \bar{B}_{i}(t)
\end{array}\right]\left[\begin{array}{l}
\xi \\
v
\end{array}\right] \\
& +\underbrace{\left[\left(\frac{\partial f_{i}}{\partial \xi}\left(\xi_{i}^{*}, v_{i}^{*}, t\right)-\bar{A}_{i}(t)\right)\left(\frac{\partial f_{i}}{\partial v}\left(\xi_{i}^{*}, v_{i}^{*}, t\right)-\bar{B}_{i}(t)\right)\right]\left[\begin{array}{c}
\xi \\
v
\end{array}\right]}, \\
& b_{i}(\xi, v, t)
\end{aligned}
$$

where $\bar{A}_{i}$ and $\bar{B}_{i}$ are the $i$-th rows of $\bar{A}$ and $\bar{B}$, respectively, and $b_{i}$ is the $i$-th output of $b$. Now let $Q_{i} \triangleq \partial f_{i} / \partial \xi$. The function $Q_{i}$ is continuous in $Z$ because $f$ is continuously differentiable in $Z$. Hence, by the Heine-Cantor theorem, $Q_{i}$ is uniformly continuous in $Z$. Therefore, for all $\epsilon>0$ there exists $\delta_{o}>0$ such that

$$
\|(\xi, v)\|<\delta_{o} \Longrightarrow\left\|Q_{i}(\xi, v, t)-\bar{A}_{i}(0,0, t)\right\|<\epsilon,
$$

for every $(\xi, v, t) \in Z$, since $Q_{i}(0,0, t)=\bar{A}_{i}(0,0, t)$. Using similar arguments for $\partial f_{i} / \partial v, \partial g_{i} / \partial \xi, \partial g_{i} / \partial v$, we can conclude that for all $\epsilon>0$ there exists $\delta_{O}>0$ satisfying

$$
\begin{array}{r}
\|(\xi, v)\|<\delta_{O} \\
\Longrightarrow \|(b(\xi, v, t), d(\xi, v, t)\|<\epsilon\|(\xi, v) \|,
\end{array}
$$

for every $(\xi, v, t) \in Z$.

\section{2 $\mathcal{L}_{\infty}$ Asymptotics of the Linearization Error}

Next, let us consider the LTV system defined by the matrices $\bar{A}, \bar{B}, \bar{C}, \bar{D}$ :

$$
\left\{\begin{array}{l}
\dot{\bar{\chi}}(t)=\bar{A}(t) \bar{\chi}(t)+\bar{B}(t) \bar{u}(t) \\
\bar{w}(t)=\bar{C}(t) \bar{\chi}(t)+\bar{D}(t) \bar{u}(t)
\end{array}\right.
$$

for all $t \in[0, T]$, where $\bar{\chi}(0)=\chi(0)$. The 0 -i.s. inputoutput response $\bar{G}_{0}$ and the initial state response $\bar{H}$ will be defined for this system as in Section 3. Subtracting (11) from (9),

$$
\left\{\begin{array}{l}
\dot{\tilde{\chi}}(t)=\bar{A}(t) \tilde{\chi}(t)+\bar{B}(t) \tilde{u}(t)+b(\chi(t), u(t), t), \\
\tilde{w}(t)=\bar{C}(t) \tilde{\chi}(t)+\bar{D}(t) \tilde{u}(t)+d(\chi(t), u(t), t),
\end{array}\right.
$$

where $\tilde{\chi}(t) \triangleq \chi(t)-\bar{\chi}(t), \tilde{w}(t) \triangleq w(t)-\bar{w}(t)$, and similarly $\tilde{u}(t) \triangleq u(t)-\bar{u}(t)$. Define the mapping $\varphi$ so that

$$
(\varphi(\chi, u))(t)=(b(\chi(t), u(t), t), d(\chi(t), u(t), t)) .
$$

Then the output error $\tilde{w}$ is given by

$$
\tilde{w}=\bar{G}_{0} \tilde{u}+\Omega(\varphi(\chi, u)),
$$

where $\Omega$ represents the $\mathcal{L}_{\infty}$ stable input-output response of an LTV system with state matrices $A,\left[\begin{array}{ll}I & 0\end{array}\right], C,\left[\begin{array}{ll}0 & I\end{array}\right]$. The following lemma will define the asymptotic behavior of $\varphi$ with respect to $(u, \chi(0))$; see (Altm and Barton 2017) for a proof.

Lemma 12 For all $\epsilon>0$, there exists $\delta^{*} \in(0, \epsilon)$ such that

$$
\begin{aligned}
\|u\|_{\mathcal{L}_{\infty}}+ & \|\chi(0)\|<\delta^{*} \\
& \Longrightarrow \| \varphi(\chi, u)) \|_{\mathcal{L}_{\infty}} \leq \epsilon\left(\|u\|_{\mathcal{L}_{\infty}}+\|\chi(0)\|\right) .
\end{aligned}
$$

\subsection{Necessary and Sufficient Conditions for Exponen- tial Stability}

We first assume that the 0 -i.s. linearized system is exponentially stable so that $\left\|\bar{G}_{0}^{k}\right\|_{\mathcal{L}_{\infty}} \leq \bar{M} \bar{\zeta}^{k}$ for all $k \in \mathbb{N}$, for some $\bar{M} \geq 1, \bar{\zeta} \in(0,1)$. With this, let $N \in \mathbb{N}$ such that $\bar{M} \bar{\zeta}^{N}<1$. We will need the subsequent result, which follows easily from Lipschitz continuity of $\Gamma$ (Lemma 4):

Lemma 13 There exist scalars $\delta_{\mathrm{fh}}>0$ and $L_{\mathrm{fh}} \geq 1$ so that $\left\|y_{0}\right\|_{\mathcal{L}_{\infty}}+\|\mathbf{x}(0)\|_{e_{1}}<\delta_{\text {fh }}$ implies

$$
\left\|y_{k}\right\|_{\mathcal{L}_{\infty}}<L_{\mathrm{fh}}\left(\left\|y_{0}\right\|_{\mathcal{L}_{\infty}}+\|\mathbf{x}(0)\|_{e_{1}}\right),
$$

for all $k \in\{0,1, \ldots, N-1\}$. 
Proposition 14 The nonlinear system (1) is exponentially stable if its linearization (10) is exponentially stable.

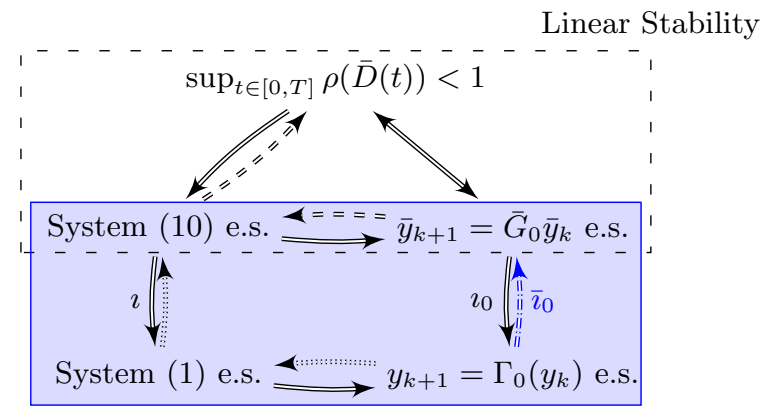

Fig. 2. Implication diagram for exponential stability (e.s.): The linear exponential stability diagram was stated in Theorem 10, where the dashed implication arrows were established by proving the solid ones. For the nonlinear case, implications $\imath, \imath_{0}$ are proven in Proposition 14. Proving implication $\bar{\imath}_{0}$ will close the loop and allow us to conclude the dotted implication arrows.

The proof of this proposition is rather involved and as such given in Appendix A for a more compact presentation. To establish the converse of this result, we will follow an indirect route that is much easier compared to a direct proof. Specifically, we will show that nonlinear exponential stability implies linear exponential stability for the 0 -i.s case. Since the 0 -i.s. systems given by the operators $\Gamma_{0}(.) \triangleq \pi_{y}(\Gamma(., 0))$ and $G_{0}$ are in essence discrete systems evolving on $\mathcal{Y}$, we will be relying on the following forward Lyapunov theorem. This will allow us to finalize our main result by aid of Theorem 10, as can be seen in Fig. 2. For obvious reasons, a functional satisfying the conditions of Theorem 15 will be called a Lyapunov functional.

Theorem 15 Let $F_{0} \in\left\{\Gamma_{0}, G_{0}\right\}$. Then, the 0-i.s. $D R P$ given by the recursion $y_{k+1}=F_{0}\left(y_{k}\right)$ on $\mathcal{Y}$ is exponentially stable if and only if there exists a functional $V: \mathcal{Y} \rightarrow \mathbb{R}$ and positive scalars $c_{1}, c_{2}, c_{3}$, with $c_{2}>c_{3}$, such that $c_{1}\|y\|_{\mathcal{L}_{\infty}} \leq V(y) \leq c_{2}\|y\|_{\mathcal{L}_{\infty}}$ and $V\left(F_{0} y\right)-V(y) \leq-c_{3}\|y\|_{\mathcal{L}_{\infty}}$ in a neighborhood of the origin.

PROOF. Sufficiency is obvious and is therefore omitted. The necessity part can be proven by construction as follows: Assume that the system is exponentially stable, then there exists $K>1, \delta_{3}>0$ and $\gamma \in[0,1)$ so that $\left\|F_{0}^{k}(y)\right\|_{\mathcal{L}_{\infty}} \leq K \gamma^{k}\|y\|_{\mathcal{L}_{\infty}}$ holds for all $y \in \mathcal{Y}$ with $\|y\|<\delta_{3}$. Let $N$ be an integer so $K \gamma^{N}<1$. Then, it is easy to show $V(y) \triangleq \sum_{i=0}^{N-1}\left\|F_{0}^{i}(y)\right\|_{\mathcal{L}_{\infty}} \geq\|y\|_{\mathcal{L}_{\infty}}$ satisfies the conditions of the theorem for all $y \in \mathcal{Y}$ with $\|y\|_{\mathcal{L}_{\infty}}<\delta_{3}$.

Proposition 16 The linearization (10) of the nonlinear system is 0-i.s. exponentially stable if the nonlinear system (1) is 0-i.s.exponentially stable.

PROOF. Let $F_{0}=\Gamma_{0}$, and let $V$ be the Lyapunov functional from the proof of Theorem 15. Then, $V$ satisfies $c_{1}\|y\|_{\mathcal{L}_{\infty}} \leq V(y) \leq c_{2}\|y\|_{\mathcal{L}_{\infty}}$, and the difference of $V$ with respect to the linear operator $\bar{G}_{0}$ is

$$
\begin{aligned}
\Delta V(y) & \triangleq V\left(\bar{G}_{0} y\right)-V(y) \\
& =\left(V\left(\bar{G}_{0} y\right)-V\left(\Gamma_{0}(y)\right)\right)+\left(V\left(\Gamma_{0}(y)\right)-V(y)\right) \\
& \leq\left(V\left(\bar{G}_{0} y\right)-V\left(\Gamma_{0}(y)\right)\right)-c_{3}\|y\|_{\mathcal{L}_{\infty}},
\end{aligned}
$$

around the origin for some positive $c_{1}, c_{2}, c_{3}$ with $c_{2}>c_{3}$, as the nonlinear DRP is exponentially stable. The functional $V$ is locally Lipschitz because it is a sum of locally Lipschitz functionals; $\Gamma_{0}^{i}$ is locally Lipschitz for any $i \in \mathbb{N}$ by Lemma 4 . Furthermore, from (12),

$$
\bar{G}_{0} y-\Gamma_{0}(y)=\Omega\left(\varphi\left(\Gamma_{x}(y, 0), y\right)\right)
$$

Recall that $\Omega$ is $\mathcal{L}_{\infty}$ stable, and $\Gamma_{x}$ is locally Lipschitz. Hence, for any $\epsilon>0$, by Lemma 12, there exists $\delta^{*}>0$ so $\|y\|_{\mathcal{L}_{\infty}}<\delta^{*}$ implies $\left|V\left(\Gamma_{0}(y)\right)-V\left(\bar{G}_{0} y\right)\right| \leq \epsilon\|y\|_{\mathcal{L}_{\infty}}$, and therefore for any $\bar{c}_{3} \in\left(0, c_{3}\right)$, there exists a $\bar{\delta}_{3}>0$ so that $\|y\|_{\mathcal{L}_{\infty}} \leq \bar{\delta}_{3}$ implies

$\Delta V(y) \leq\left(V\left(\bar{G}_{0} y\right)-V\left(\Gamma_{0}(y)\right)\right)-c_{3}\|y\|_{\mathcal{L}_{\infty}} \leq \bar{c}_{3}\|y\|_{\mathcal{L}_{\infty}}$.

By Theorem 15, it follows that the linearization (10) is 0 -i.s. exponentially stable.

We are now ready to state our main result, which summarizes the findings of Theorem 10 and Propositions 14 and 16 as given below:

Theorem 17 For the nonlinear DRP (1) and its linearization (10), the following are equivalent:

(1) The DRP (1) is exponentially stable.

(2) The 0-i.s. DRP (1) is exponentially stable.

(3) The DRP (10) is globally exponentially stable.

(4) The 0-.i.s. DRP (10) is globally exponentially stable.

(5) The condition $\max _{t \in[0, T]} \rho(\bar{D}(t))<1$ holds.

Theorem 17 is an important result that can be readily applied to many existing algorithms. For example, it can be shown that Picard iterates form an exponentially stable DRP (Altin and Barton 2017), and therefore have nonzero robustness with respect to initial condition errors. In the next section, the application of this result to stability analysis of ILC will be discussed in depth. 


\section{Application: Exponential Stability of ILC}

This section will address the application of Theorem 17 to the ILC problem of iteratively constructing the feedforward input $u^{*}$ given a desired output $y_{\text {des }}$ so that

$$
\left\{\begin{aligned}
\dot{x}^{*}(t) & =f\left(x^{*}(t), u^{*}(t), t\right), \\
y_{\mathrm{des}}(t) & =g\left(x^{*}(t), u^{*}(t), t\right)
\end{aligned}\right.
$$

for all $t \in[0, T]$. We consider the ILC system, where the continuously differentiable function $l$ satisfies $l(0, t)=0$,

$$
\left\{\begin{array}{l}
\dot{x}_{k+1}(t)=f\left(x_{k+1}(t), u_{k+1}(t), t\right), \\
y_{k+1}(t)=g\left(x_{k+1}(t), u_{k+1}(t), t\right), \\
u_{k+1}(t)=u_{k}(t)+l\left(e_{k}(t), t\right),
\end{array}\right.
$$

and $e_{k} \triangleq y_{k}-y_{\text {des }}$, for all $(t, k) \in[0, T] \times \mathbb{N}$. This static (in-time) update law is based on the internal model principle in the iteration domain, and guarantees perfect tracking in the limit for all achievable $y_{\text {des }}$ when stable. Following a time-varying coordinate transformation shifting the equilibrium to the origin, details of which are skipped, we can rewrite the system as

$$
\left\{\begin{aligned}
\dot{x}_{k+1}(t) & =\underline{f}\left(\underline{x}_{k+1}(t), \underline{u}_{k}(t), e_{k}(t), t\right), \\
{\left[\begin{array}{c}
e_{k+1}(t) \\
\underline{u}_{k+1}(t)
\end{array}\right] } & =\left[\begin{array}{c}
\underline{g}\left(\underline{x}_{k+1}(t), \underline{u}_{k}(t), e_{k}(t), t\right), \\
\underline{u}_{k}(t)+l\left(e_{k}(t), t\right)
\end{array}\right],
\end{aligned}\right.
$$

with

$\underline{g}(\chi, u, \theta, t) \triangleq g\left(\chi+x^{*}(t), u+u^{*}(t)+l(\theta, t), t\right)-y_{\mathrm{des}}(t)$,

for all $(t, k) \in[0, T] \times \mathbb{N}$. Observe that $e_{0}$ depends on $\underline{u}_{0}$, so $\left(e_{0}, \underline{u}_{0}\right)$ cannot be arbitrarily chosen, and thus it is difficult to derive necessary stability conditions. Nevertheless, letting

$$
\underline{D}(t) \triangleq \frac{\partial g}{\partial u}\left(x^{*}(t), u^{*}(t), t\right), \quad \underline{L}(t) \triangleq \frac{\partial l}{\partial \theta}(0, t),
$$

for all $t \in[0, T]$, the system is exponentially stable if

$$
\max _{t \in[0, T]} \rho\left(\left[\begin{array}{c}
\underline{D}(t) \\
I
\end{array}\right][\underline{L}(t) I]\right)=\max _{t \in[0, T]} \rho(I+\underline{L}(t) \underline{D}(t))<1,
$$

where the equality can be verified via simple eigenvector manipulations, with the equivalent condition being $\max _{t \in[0, T]} \rho(I+\underline{D}(t) \underline{L}(t))<1$ for square systems. Note that the same methodology can be used to derive spectral stability conditions with $Q$ filtering; i.e. the update is of the form $u_{k+1}(t)=\underline{Q}(t) u_{k}(t)+l\left(e_{k}(t), t\right)$, a known robust stabilization factor in ILC algorithms.
The stability result derived above is the first eigenvalue based condition in the nonlinear ILC literature. Its significance further stems from the fact that it unifies several important results, such as continuous dependence of the tracking error on initial condition errors (Heinzinger et al. 1992), and the principle that the error term in the function $l$ must be replaced with its $\bar{n}$-th derivative for a relative degree $\bar{n}$ system (Ahn et al. 1993)). Furthermore, it is among the first studies of ILC from a local perspective, which enables nonlinear time-varying update laws to be considered without resorting to saturation (Tan et al. 2015), and provides a rigorous basis to linearization in the context of ILC (Bristow et al. 2006).

\section{Illustrative Example}

Consider the actuated Van der Pol oscillator in normal form with a time-varying damping coefficient:

$$
\left\{\begin{aligned}
\dot{q}_{1}(t) & =q_{2}(t), \\
\dot{q}_{2}(t) & =-q_{1}(t)+\Xi(t)\left(1-\left(q_{1}(t)\right)^{2}\right) q_{2}(t)+u(t), \\
y & =q_{1}(t),
\end{aligned}\right.
$$

where the damping coefficient $\Xi(t)>0$, and $t \in[0,2]$. The unforced oscillator is well known to have an unstable equilibrium at the origin for all constant $\Xi(t)>0$. Our objective is to find an ILC update law in order to track the reference $y_{\mathrm{des}}(t)=0.1 \cos (2 \pi t)$. Since the relative degree is 2 , we consider the update

$u_{k+1}(t)=u_{k}(t)-\left(\ddot{y}_{k}(t)-\ddot{y}_{\text {des }}(t)\right), \quad \forall(t, k) \in[0,2] \times \mathbb{N}$.

Then, it is easy to verify that this update law is stable since $\ddot{y}(t)=\dot{q}_{2}(t)$ and $\left(\partial \dot{q}_{2} / \partial u\right)(t)=1$. Indeed, for $\Xi(t)=4+0.5 \sin (2 \pi(10 t))$, Fig. 3 shows that the tracking error is exponentially decreased when $u_{0}=0$ and the initial conditions are randomly chosen to exponentially converge to $\left(y_{\text {des }}(0), \dot{y}_{\text {des }}(0)\right)=(0.1,0)$ with convergence rate $\lambda$ (also randomly chosen) and $e_{\lambda}$ norm less than 0.1, without any stabilizing feedback.

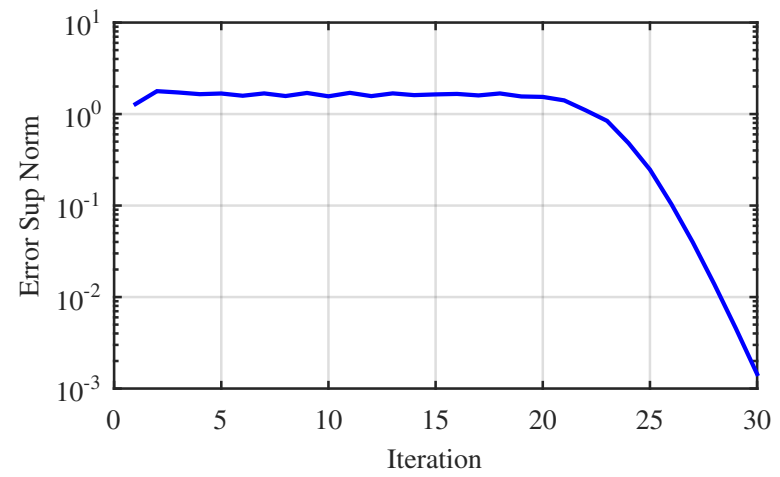

Fig. 3. Evolution of $\left\|e_{k}\right\|_{\mathcal{L}_{\infty}}$. 


\section{Conclusion}

This paper addressed the problem of finding necessary and sufficient exponential stability conditions for a class of nonlinear repetitive processes and showed that a DRP is exponentially stable if and only if the state matrix $\bar{D}$ of its linearization is uniformly Schur over the time interval $[0, T]$. To our knowledge, the work presented here is the first systematic study of local stability for nonlinear repetive processes. The findings of the paper are especially important since local stability is the precursor to global stability. The comprehensiveness of these results are reflected in the fact that they tie in the various existing results from nonlinear ILC analysis via a single framework. We hope that the analysis presented in the paper will pave the way for further research on nonlinear repetitive processes and other 2D systems, such as extensions to different classes of systems and the corresponding control strategies.

\section{A Proof of Proposition 14}

By (12), the output at pass $k+1$ can be written as

$$
y_{k+1}=\bar{H} x_{k+1}(0)+\bar{G}_{0} y_{k}+\Omega\left(\varphi\left(x_{k+1}, y_{k}\right)\right),
$$

so

$$
y_{k}=\bar{G}_{0}^{k} y_{0}+\sum_{i=1}^{k} \bar{G}_{0}^{k-i}\left(\bar{H} x_{i}(0)+\Omega\left(\varphi\left(x_{i}, y_{i-1}\right)\right)\right)
$$

for all $k \in \mathbb{N}$, when the solution exists. Recalling the fact that $\left\|\bar{G}_{0}^{k}\right\|_{\mathcal{L}_{\infty}} \leq \bar{M} \bar{\zeta}^{k}$ for all $k \in \mathbb{N}$ for some $\bar{M} \geq 1$ and $\bar{\zeta} \in(0,1)$, from (A.1), it follows that

$$
\begin{gathered}
\left\|y_{N}\right\|_{\mathcal{L}_{\infty}} \leq \bar{M} \bar{\zeta}^{N}\left\|y_{0}\right\|_{\mathcal{L}_{\infty}}+\max \left\{\|\bar{H}\|_{\mathcal{L}_{\infty}},\|\Omega\|_{\mathcal{L}_{\infty}}\right\} \\
\times\left(\|\mathbf{x}(0)\|_{e_{1}}+\max _{i \in\{1,2, \ldots, N\}}\left\|\varphi\left(x_{i}, y_{i-1}\right)\right\|_{\mathcal{L}_{\infty}}\right) \sum_{i=1}^{N} \bar{M} \bar{\zeta}^{N-i}
\end{gathered}
$$

therefore

$$
\begin{aligned}
&\left\|y_{N}\right\|_{\mathcal{L}_{\infty}} \leq \underbrace{\bar{M} \bar{\zeta}^{N}}_{r_{1}<1}\left\|y_{0}\right\|_{\mathcal{L}_{\infty}} \\
&+\underbrace{\bar{M} \frac{1-\bar{\zeta}^{N}}{1-\bar{\zeta}} \max \left\{\|\bar{H}\|_{\mathcal{L}_{\infty}},\|\Omega\|_{\mathcal{L}_{\infty}}\right\}}_{r_{2}>0} \\
& \times\left(\|\mathbf{x}(0)\|_{e_{1}}+\max _{i \in\{1,2, \ldots, N\}}\left\|\varphi\left(x_{i}, y_{i-1}\right)\right\|_{\mathcal{L}_{\infty}}\right) .
\end{aligned}
$$

The rest of the proof will be divided into three steps:

\section{A.1 Lyapunov Stability}

This part follows the same basic ideas of (Altin and Barton 2015, Lemma 3). Take any $\epsilon \in\left(0,\left(1-r_{1}\right) / r_{2}\right)$, where $r_{1}, r_{2}$ are defined in (A.2). By Lemmas 12 and 13, there exist $\delta^{*} \in(0, \epsilon)$ and $\delta_{\mathrm{fh}}^{*} \in\left(0, \min \left\{\delta_{\mathrm{fh}}, \delta^{*} / L_{\mathrm{fh}}\right\}\right)$ such that $\left\|y_{0}\right\|_{\mathcal{L}_{\infty}}+\|\mathbf{x}(0)\|_{e_{1}}<\delta_{\mathrm{fh}}^{*}<\delta^{*} / L_{\mathrm{fh}}<\delta^{*}$ means

$$
\left\|y_{k}\right\|_{\mathcal{L}_{\infty}} \leq L_{\mathrm{fh}}\left(\left\|y_{0}\right\|_{\mathcal{L}_{\infty}}+\|\mathbf{x}(0)\|_{e_{1}}\right)<\delta^{*}<\epsilon
$$

for all $k \in\{0,1, \ldots, N-1\}$, which in turn implies

$$
\begin{aligned}
\left\|\varphi\left(x_{k}, y_{k-1}\right)\right\|_{\mathcal{L}_{\infty}} & <\epsilon /\left(L_{\mathrm{fh}}+1\right)\left(\left\|y_{k-1}\right\|_{\mathcal{L}_{\infty}}+\|\mathbf{x}(0)\|_{e_{1}}\right) \\
& <\epsilon\left(\left\|y_{0}\right\|_{\mathcal{L}_{\infty}}+\|\mathbf{x}(0)\|_{e_{1}}\right)
\end{aligned}
$$

for all $k \in\{1,2, \ldots, N\}$. Assume $\left\|y_{0}\right\|_{\mathcal{L}_{\infty}}<\delta_{y} \leq \delta_{\text {fh }}^{*} / 2$ and $\|\mathbf{x}(0)\|_{e_{1}}<\delta_{x} \leq r_{y} \delta_{y}$ for arbitrary $r_{y}$ satisfying

$$
r_{y} \in\left(0, \min \left\{1, \frac{1-r_{1}-r_{2} \epsilon}{r_{2}(1+\epsilon)}\right\}\right) .
$$

The interval above is nonempty since $\epsilon<\left(1-r_{1}\right) / r_{2}$, and if $r_{y}$ belongs to this interval, $\delta_{x}+\delta_{y}<2 \delta_{y} \leq \delta_{\mathrm{fh}}^{*}$. It follows by the above arguments and (A.2) that

$$
\begin{aligned}
& \left\|y_{N}\right\|_{\mathcal{L}_{\infty}} \leq r_{1}\left\|y_{0}\right\|_{\mathcal{L}_{\infty}} \\
& +r_{2}\left(\|\mathbf{x}(0)\|_{e_{1}}+\epsilon\left(\left\|y_{0}\right\|_{\mathcal{L}_{\infty}}+\|\mathbf{x}(0)\|_{e_{1}}\right)\right) \\
& \quad \leq\left\|y_{0}\right\|_{\mathcal{L}_{\infty}}\left(r_{1}+r_{2} \epsilon\right)+\|\mathbf{x}(0)\|_{e_{1}} r_{2}(1+\epsilon)
\end{aligned}
$$

so $\left\|y_{N}\right\|_{\mathcal{L}_{\infty}} \leq \delta_{y}\left(r_{1}+r_{2} \epsilon\right)+\delta_{x} r_{2}(1+\epsilon)=r_{N} \delta_{y}<\delta_{y}$, where $r_{N} \triangleq\left(r_{1}+r_{2} \epsilon\right)+r_{y} r_{2}(1+\epsilon)<1$. Moreover, by (A.3), $\left\|y_{k}\right\|_{\mathcal{L}_{\infty}}<\epsilon$ for all $k \in\{1,2, \ldots, N-1\}$. By induction, $\left\|y_{0}\right\|_{\mathcal{L}_{\infty}}<\delta_{y}$ and $\|\mathbf{x}(0)\|_{e_{1}}<\delta_{x}$ implies $\left\|y_{k}\right\|_{\mathcal{L}_{\infty}}<\epsilon$ for all $k \in \mathbb{N}$, since $\delta_{y}<\epsilon$. Therefore, if $\left\|y_{0}\right\|_{\mathcal{L}_{\infty}}+\|\mathbf{x}(0)\|<\delta_{1}=\min \left\{\delta_{x}, \delta_{y}\right\}$, then $\left\|y_{k}\right\|_{\mathcal{L}_{\infty}}<\epsilon$ for all $k \in \mathbb{N}$. As we can find such a $\delta_{1}>0$ for arbitrarily small $\epsilon>0$, we conclude that the system is stable.

\section{A.2 Asymptotic Stability}

From (A.1), $y_{k}=\bar{y}_{k}+\sum_{i=1}^{k} \bar{G}_{0}^{k-i} \Omega\left(\varphi\left(x_{i}, y_{i-1}\right)\right)$. Let $\epsilon=(1-\bar{\zeta}) /\left(2 \bar{M}\|\Omega\|_{\mathcal{L}_{\infty}}\right)$. Since the system is stable, by Lemma 12 there exists a positive scalar $\delta_{1}$ so that $\left\|y_{0}\right\|_{\mathcal{L}_{\infty}}+\left\|\mathbf{x}(0)_{e_{1}}\right\|<\delta_{2}=\delta_{1}$ implies

$$
\begin{aligned}
& \limsup _{k \rightarrow \infty}\left\|y_{k}\right\|_{\mathcal{L}_{\infty}} \\
& \leq \epsilon \bar{M}\|\Omega\|_{\mathcal{L}_{\infty}} \limsup _{k \rightarrow \infty} \sum_{i=1}^{k} \bar{\zeta}^{k-i}\left(\left\|x_{i}(0)\right\|+\left\|y_{i-1}\right\|_{\mathcal{L}_{\infty}}\right) \\
& \quad=\epsilon \bar{M}\|\Omega\|_{\mathcal{L}_{\infty}} \limsup _{k \rightarrow \infty} \underbrace{\sum_{i=1}^{k} \bar{\zeta}^{k-i}\left\|y_{i-1}\right\|_{\mathcal{L}_{\infty}}}_{\bar{S}_{k}},
\end{aligned}
$$


as $\bar{y}_{k} \rightarrow 0$, and $\sum_{i=1}^{k} \bar{\zeta}^{k-i}\left\|x_{i}(0)\right\| \rightarrow 0$ if $\mathbf{x}(0) \in c_{0}$, as we have shown before in Section 3 . Now, it is easy to verify that $\bar{S}_{k+1}=\bar{\zeta} \bar{S}_{k}+\left\|y_{k}\right\|_{\mathcal{L}_{\infty}}$, where $\bar{S}_{k}$ is defined in (A.4). Hence by (A.4) and Claim 1 we can show

$$
\limsup _{k \rightarrow \infty}\left\|y_{k}\right\|_{\mathcal{L}_{\infty}} \leq \frac{1}{2} \limsup _{k \rightarrow \infty}\left\|y_{k}\right\|_{\mathcal{L}_{\infty}}
$$

so $\lim \sup _{k \rightarrow \infty}\left\|y_{k}\right\|_{\mathcal{L}_{\infty}}=\lim _{k \rightarrow \infty}\left\|y_{k}\right\|_{\mathcal{L}_{\infty}}=0$. Therefore, the system is asymptotically stable.

\section{A.3 Exponential Stability}

Let $\mathbf{x}_{\kappa}(0) \triangleq\left\{x_{k+1}(0)\right\}_{k=\kappa}^{\infty}$ for any $\kappa \in \mathbb{N}$. As we have proved Lyapunov stability, given $\epsilon>0$, by (A.2) and Lemmas 12 and 13 , we can find a constant $\delta_{3} \in\left(0, \delta_{\mathrm{fh}}\right)$ such that $\left\|y_{0}\right\|_{\mathcal{L}_{\infty}}+\|\mathbf{x}(0)\|_{e_{\lambda}}<\delta_{3}$ implies $\left\|y_{k}\right\|_{\mathcal{L}_{\infty}}<\delta_{\text {fh }}$ and

$$
\begin{aligned}
& \left\|y_{(k+1) N}\right\|_{\mathcal{L}_{\infty}} \leq r_{1}\left\|y_{k N}\right\|_{\mathcal{L}_{\infty}} \\
& +r_{2}\left(\lambda^{k N}\|\mathbf{x}(0)\|_{e_{\lambda}}+\epsilon\left(\left\|y_{k N}\right\|_{\mathcal{L}_{\infty}}+\lambda^{k N}\|\mathbf{x}(0)\|_{e_{\lambda}}\right)\right) \\
& \quad \leq\left(r_{1}+r_{2} \epsilon\right)\left\|y_{k N}\right\|_{\mathcal{L}_{\infty}}+r_{2}(1+\epsilon)\|\mathbf{x}(0)\|_{e_{\lambda}} \lambda^{k N}
\end{aligned}
$$

where we use the $e_{\lambda}$ norm shift and $\lambda$ properties, and $r_{1}, r_{2}$ are defined in (A.2); hence,

$$
\begin{aligned}
& \left\|y_{k N}\right\|_{\mathcal{L}_{\infty}} \leq\left(r_{1}+r_{2} \epsilon\right)^{k}\left\|y_{0}\right\|_{\mathcal{L}_{\infty}} \\
& \quad+r_{2}(1+\epsilon)\|\mathbf{x}(0)\|_{e_{\lambda}} \sum_{i=1}^{k}\left(r_{1}+r_{2} \epsilon\right)^{k-i}\left(\lambda^{N}\right)^{i-1}
\end{aligned}
$$

for all $k \in \mathbb{N}$. Now take any

$$
\epsilon \in\left(\max \left\{0, \frac{1-r_{1}-2 r_{2}}{3 r_{2}}\right\}, \frac{1-r_{1}}{r_{2}}\right) .
$$

Then, $r_{1}+r_{2} \epsilon<1$. Letting $\underline{\lambda}_{N} \triangleq \max \left\{r_{1}+r_{2} \epsilon, \lambda^{N}\right\}$, as before in the linear case of Section 3.3, we can find continuous increasing functions

$$
\begin{aligned}
& K_{N}\left(\lambda^{N}\right) \triangleq \max \left\{1, \frac{2 r_{2}(1+\epsilon)}{1-\underline{\lambda}_{N}}\right\}=\frac{2 r_{2}(1+\epsilon)}{1-\underline{\lambda}_{N}}, \\
& \gamma_{N}\left(\lambda^{N}\right) \triangleq \frac{1+\underline{\lambda}_{N}}{2} \in\left[\lambda^{N}, 1\right)
\end{aligned}
$$

by Claim 2, such that $\left\|y_{0}\right\|_{\mathcal{L}_{\infty}}+\|\mathbf{x}(0)\|_{e_{\lambda}}<\delta_{3}$ implies

$$
\left\|y_{k N}\right\|_{\mathcal{L}_{\infty}} \leq K_{N}\left(\lambda^{N}\right) \gamma_{N}\left(\lambda^{N}\right)^{k}\left(\left\|y_{0}\right\|_{\mathcal{L}_{\infty}}+\|\mathbf{x}(0)\|_{e_{\lambda}}\right),
$$

and since $\left\|y_{k}\right\|_{\mathcal{L}_{\infty}} \leq \delta_{\text {fh }}$ for all $k \in \mathbb{N}$, by Lemma 13 and the $e_{\lambda}$ shift property,

$$
\begin{array}{r}
\left\|y_{k}\right\|_{\mathcal{L}_{\infty}} \leq L_{\mathrm{fh}} K_{N}\left(\lambda^{N}\right) \gamma_{N}\left(\lambda^{N}\right)^{\bar{k}}\left(\left\|y_{0}\right\|_{\mathcal{L}_{\infty}}+\|\mathbf{x}(0)\|_{e_{\lambda}}\right) \\
+L_{\mathrm{fh}}\left(\lambda^{N}\right)^{\bar{k}}\|\mathbf{x}(0)\|_{e_{\lambda}},
\end{array}
$$

for all $k \in \mathbb{N}$ as $L_{\mathrm{fh}} \geq 1$, where $\bar{k} \in \mathbb{N}$ satisfies $k=\bar{k} N+j$ and $j \in\{0,1, \ldots, N-1\}$. In turn, this means that

$$
\left\|y_{k}\right\|_{\mathcal{L}_{\infty}} \leq 2 L_{\mathrm{fh}} K_{N}\left(\lambda^{N}\right) \gamma_{N}\left(\lambda^{N}\right)^{\bar{k}}\left(\left\|y_{0}\right\|_{\mathcal{L}_{\infty}}+\|\mathbf{x}(0)\|_{e_{\lambda}}\right),
$$

for all $k \in \mathbb{N}$. Let $\gamma(\lambda) \triangleq\left(\gamma_{N}\left(\lambda^{N}\right)\right)^{1 / N}$. Then,

$$
\left\|y_{k}\right\|_{\mathcal{L}_{\infty}} \leq 2 L_{\mathrm{fh}} K_{N}\left(\lambda^{N}\right) \gamma(\lambda)^{k-j}\left(\left\|y_{0}\right\|_{\mathcal{L}_{\infty}}+\|\mathbf{x}(0)\|_{e_{\lambda}}\right)
$$

hence, as $\gamma(\lambda) \in(0,1)$ and $j \leq N-1$,

$$
\begin{aligned}
\left\|y_{k}\right\|_{\mathcal{L}_{\infty}} \leq \overbrace{2 L_{\mathrm{fh}} K_{N}\left(\lambda^{N}\right) \gamma(\lambda)^{1-N}}^{K(\lambda)} \gamma(\lambda)^{k} \\
\left(\left\|y_{0}\right\|_{\mathcal{L}_{\infty}}+\|\mathbf{x}(0)\|_{e_{\lambda}}\right),
\end{aligned}
$$

for all $k \in \mathbb{N}$. Clearly, $\gamma$ is continuous and increasing as before, while $K$ defined in (A.5) is continuous. It remains to show that $K$ is increasing. Since

$$
\begin{aligned}
K(\lambda) & =2 L_{\mathrm{fh}} \frac{K_{N}\left(\lambda^{N}\right)}{\gamma(\lambda)^{N}} \gamma(\lambda)=2 L_{\mathrm{fh}} \frac{K_{N}\left(\lambda^{N}\right)}{\gamma_{N}\left(\lambda^{N}\right)} \gamma(\lambda) \\
& =2 L_{\mathrm{fh}} \frac{2 r_{2}(1+\epsilon)}{1-\underline{\lambda}_{N}} \frac{2}{1+\underline{\lambda}_{N}} \gamma(\lambda) \\
& =8 L_{\mathrm{fh}} r_{2}(1+\epsilon) \frac{\gamma(\lambda)}{1-\underline{\lambda}_{N}^{2}},
\end{aligned}
$$

it follows that $K$ is increasing, as $\left(1-\underline{\lambda}_{N}^{2}\right)^{-1}$ is increasing on $(0,1)$ as a function of $\underline{\lambda}_{N}$.

\section{Acknowledgements}

This work was conducted while the first author was with the Department of Electrical Engineering and Computer Science at the University of Michigan, and supported by the NSF grant CMMI-1334204.

\section{References}

Ahn, Hyo-Sung, Yang-Quan Chen and K.L. Moore (2007) Iterative learning control: Brief survey and categorization. Systems, Man, and Cybernetics, Part C: Applications and Reviews, IEEE Transactions on 37(6), 1099-1121.

Ahn, Hyun-Sik, Chong-Ho Choi and Kwang-Bae Kim (1993). Iterative learning control for a class of nonlinear systems. Automatica 29(6), 1575 - 1578.

Albertos, Pedro and Sala, Antonio, Eds.) (2002). Iterative Identification and Control. Springer-Verlag. London.

Altin, B. and K. Barton (2015). On linearized stability of differential repetitive processes and iterative learning control. In: Decision and Control (CDC), 2015 IEEE 54th Annual Conference on. pp. 6064-6069.

Altın, Berk and Kira Barton (2017). Exponential Stability of Nonlinear Differential Repetitive Processes with Applications to Iterative Learning Control. ArXiv e-prints. 
Barreiro, Antonio and Alfonso Baos (2010). Delay-dependent stability of reset systems. Automatica 46(1), $216-221$.

Bristow, D.A., M. Tharayil and A.G. Alleyne (2006). A survey of iterative learning control. Control Systems, IEEE 26(3), 96114 .

Chesi, G. and R.H. Middleton (2014). Necessary and sufficient lmi conditions for stability and performance analysis of 2-D mixed continuous-discrete-time systems. Automatic Control, IEEE Transactions on 59(4), 996-1007.

Chesi, G. and R.H. Middleton (2015). $\mathcal{H}_{\infty}$ and $\mathcal{H}_{2}$ norms of 2-D mixed continuous-discrete-time systems via rationallydependent complex Lyapunov functions. Automatic Control, IEEE Transactions on 60(10), 2614-2625.

Devasia, S., Degang Chen and B. Paden (1996). Nonlinear inversion-based output tracking. IEEE Transactions on Automatic Control 41(7), 930-942.

Edwards, J. B. and D. H. Owens (1982). Analysis and Control of Multipass Processes. John Wiley \& Sons. New York, NY.

Edwards, J.B. (1974). Stability problems in the control of multipass processes. Electrical Engineers, Proceedings of the Institution of 121(11), 1425-1432.

Emelianov, Mikhail, Pavel Pakshin, Krzysztof Galkowski and Eric Rogers (2014). Stability and stabilization of differential nonlinear repetitive processes with applications. In: 19th IFAC World Congress, 2014. Vol. 19. pp. 5467-5472.

Foda, S. and P. Agathoklis (1992). Control of the metal rolling process: A multidimensional system approach. Journal of the Franklin Institute 329(2), 317 - 332.

Fornasini, E. and G. Marchesini (1976). State-space realization theory of two-dimensional filters. IEEE Transactions on Automatic Control 21(4), 484-492.

Fornasini, E. and G. Marchesini (1978). Doubly-indexed dynamical systems: State-space models and structural properties. Mathematical systems theory 12(1), 59-72.

Gupta, R., J.S. Hudson, A.M. Bloch and I.V. Kolmanovsky (2013). Optimal control of manifold filling during vde mode transitions. In: Decision and Control (CDC), 2013 IEEE 52nd Annual Conference on. pp. 2227-2232.

Heinzinger, Greg, D. Fenwick, B. Paden and F. Miyazaki (1992). Stability of learning control with disturbances and uncertain initial conditions. Automatic Control, IEEE Transactions on 37(1), 110-114.

Hladowski, Lukasz, Krzysztof Galkowski, Zhonglun Cai, Eric Rogers, Chris T. Freeman and Paul L. Lewin (2010). Experimentally supported 2D systems based iterative learning control law design for error convergence and performance. Control Engineering Practice 18(4), 339 - 348.

Kurek, J. E. and M. B. Zaremba (1993). Iterative learning control synthesis based on 2-D system theory. IEEE Transactions on Automatic Control 38(1), 121-125.

Liu, J. and A. R. Teel (2016). Lyapunov-based sufficient conditions for stability of hybrid systems with memory. IEEE Transactions on Automatic Control 61(4), 1057-1062.

Moore, K.L., Yang-Quan Chen and Hyo-Sung Ahn (2006). Iterative learning control: A tutorial and big picture view. In: Decision and Control, 2006 45th IEEE Conference on. pp. 2352-2357.

Pakshin, P., K. Galkowski and E. Rogers (2011). Stability and stabilization of systems modeled by $2 \mathrm{D}$ nonlinear stochastic Roesser models. In: Multidimensional ( $n D)$ Systems ( $n D s)$, 2011 7th International Workshop on. pp. 1-5.
Przyluski, K.Maciej (1980). The Lyapunov equation and the problem of stability for linear bounded discrete-time systems in Hilbert space. Applied Mathematics and Optimization 6(1), $97-112$.

Roesser, R. (1975). A discrete state-space model for linear image processing. IEEE Transactions on Automatic Control 20(1), 1-10.

Rogers, Eric, Krzysztof Galkowski and David H. Owens (2007). Control Systems Theory and Applications for Linear Repetitive Processes. Springer-Verlag. Berlin.

Sammons, Patrick M., Douglas A. Bristow and Robert G. Landers (2013). Height dependent laser metal deposition process modeling. Journal of Manufacturing Science and Engineering 135(5), 054501:1-7.

Sammons, Patrick M., Douglas A. Bristow and Robert G. Landers (2014). Repetitive process control of laser metal deposition. In: ASME 2014 Dynamic Systems and Control Conference. Vol. 2.

Sun, Ye, A. N. Michel and Guisheng Zhai (2005). Stability of discontinuous retarded functional differential equations with applications. IEEE Transactions on Automatic Control $\mathbf{5 0}(8), 1090-1105$.

Tan, Y., S.P. Yang and J.X. Xu (2015). On P-type iterative learning control for nonlinear systems without global Lipschitz continuity condition. In: American Control Conference (ACC), 2015. pp. 3552-3557.

Vidyasagar, Mathukumalli (2002). Input-Output Stability. Chap. 6, pp. 270-375. Society for Industrial and Applied Mathematics. Philedelphia, PA.

Weiss, George (1989). Weakly $\ell_{p}$ stable linear operators are power stable. International Journal of Systems Science 20(11), 2323-2328.

$\mathrm{Xu}$, Jian-Xin (2011). A survey on iterative learning control for nonlinear systems. International Journal of Control 84(7), 1275-1294.

Yeganefar, N., N. Yeganefar, M. Ghamgui and E. Moulay (2013). Lyapunov theory for 2-D nonlinear Roesser models: Application to asymptotic and exponential stability. Automatic Control, IEEE Transactions on 58(5), 1299-1304.

Zidek, R.A.E. and I.V. Kolmanovsky (2015). Approximate optimal control of nonlinear systems with quadratic performance criteria. In: American Control Conference (ACC), 2015. pp. 5587-5592. 